2000]53C27, 55N22, 57R65, 58J05, 58J50

\title{
SURGERY AND THE SPECTRUM OF THE DIRAC OPERATOR
}

\author{
CHRISTIAN BÄR AND MATTIAS DAHL
}

\begin{abstract}
We show that for generic Riemannian metrics on a simply-connected closed spin manifold of dimension $\geq 5$ the dimension of the space of harmonic spinors is no larger than it must be by the index theorem. The same result holds for periodic fundamental groups of odd order.

The proof is based on a surgery theorem for the Dirac spectrum which says that if one performs surgery of codimension $\geq 3$ on a closed Riemannian spin manifold, then the Dirac spectrum changes arbitrarily little provided the metric on the manifold after surgery is chosen properly.
\end{abstract}

\section{INTRODUCTION}

Classical Hodge-deRham theory establishes a tight link between the analysis of the Laplace operator acting on differential forms of a compact Riemannian manifold and its topology. Specifically, the dimension of the space of harmonic $k$-forms is a topological invariant, the $k^{\text {th }}$ Betti number.

The question arises whether a similar relation holds for other elliptic geometric differential operators such as the Dirac operator on a compact Riemannian spin manifold. It is not hard to see that the dimension $h_{g}$ of the space of harmonic spinors is a conformal invariant, it does not change when one replaces the Riemannian metric $g$ by a conformally equivalent one [10, Prop. 1.3]. Moreover, the Atiyah-Singer index theorem implies a topological lower bound on $h_{g}$.

Berger metrics on spheres of dimension $4 k+3$ provide examples showing that in general $h_{g}$ depends on the metric and is not topological, see [10, Prop. 3.2] and [3, Thm. 3.1]. Also for surfaces of genus at least 3 the number $h_{g}$ varies with the choice of metric [10, Thm. 2.6]. All known examples indicate that the following two conjectures should be true. On the one hand, we should have

Conjecture A. Harmonic spinors are not topologically obstructed, i.e. on any compact spin manifold of dimension at least three there is a metric $g$ such that $h_{g}>0$.

Perhaps $h_{g}$ is even unbounded. Conjecture A has been shown to be true in dimensions $n \equiv 0,1,3 \bmod 8$ by Hitchin [10, Thm. 4.5] using a topological approach, while the first author [3, Thm. A] proves it for $n \equiv 3,7 \bmod 8$ using an analytic approach. Conjecture A has been shown to hold for spheres of dimension $n \equiv 0 \bmod 4$ with unbounded $h_{g}$ by Seeger [23].

Date: October 22, 2018.

1991 Mathematics Subject Classification. [.

Key words and phrases. Spectrum of the Dirac operator, harmonic spinors, generic metrics, alphagenus, surgery, bordism. 
Conjecture A is not true in dimension 2. For example, on $S^{2}$ all metrics are conformally equivalent, hence $h_{g}$ cannot change with the metric. In fact, $h_{g}=0$ and one even has a simple geometric lower bound for all Dirac eigenvalues $\lambda$ on the 2-sphere equipped with any metric $\lambda^{2} \geq 4 \pi / \operatorname{area}(M)$, see [2, Thm. 2].

In this paper we do not deal with the rather exceptional metrics giving rise to large $h_{g}$ but instead we study generic metrics. Versions of the second conjecture can be found in [3, 6, 13].

Conjecture B. On any compact connected spin manifold for a generic metric $h_{g}$ is no larger than it is forced to be by the index theorem.

This means that up to a "small" set of exceptional metrics an analogue of classical Hodge-deRham theory also holds for the Dirac operator. In particular, in dimensions divisible by 4 Conjecture B claims that for a generic metric either the kernel or the cokernel of the chiral Dirac operator vanishes. Using a variational approach Maier [17] proved Conjecture B for dimension $n \leq 4$. In the present article we deal with the case of dimension $n \geq 5$. We show

Theorem 3.10. Conjecture B is true for all simply connected spin manifolds of dimension at least five.

Our approach, very different from Maier's, follows the strategy that has successfully been used to characterize manifolds admitting metrics of positive scalar curvature. We show that the class of manifolds for which Conjecture B holds is closed under surgery of codimension $\geq 3$. Then we find a set of manifolds for which the conjecture holds and which generates the spin bordism ring $\Omega_{*}^{\text {spin }}$. This set consists of manifolds of positive scalar curvature and a few more special manifolds. Results from bordism theory then imply the theorem.

Using more refined spin bordism rings one can also deal with certain classes of nonsimply connected manifolds. As an example, we show

Theorem 3.12. Suppose $M$ is a compact connected spin manifold with $\operatorname{dim} M \geq 5$ and fundamental group $\pi$ a periodic group of odd order. Then Conjecture B holds for $M$.

By exhibiting concrete examples Kotschick [13] shows that the analogue of Conjecture B does not hold in the class of Kähler manifolds.

The most involved part of our technique is to show the invariance under surgery mentioned above. To get this we show that if one performs surgery of codimension $\geq 3$ to a closed Riemannian spin manifold, then the lower part of the Dirac spectrum changes arbitrarily little provided the metric on the manifold after surgery is chosen properly. More precisely, we show

Theorem 1.2. Let $(M, g)$ be a closed Riemannian manifold equipped with a spin structure. Let $N \subset M$ be an embedded sphere of codimension $k \geq 3$ and with trivialized tubular neighborhood. Let $\widetilde{M}$ be obtained from $M$ by surgery along $N$ together with the resulting spin structure. Let $\varepsilon>0$ and $\Lambda>0, \pm \Lambda \notin \operatorname{spec}\left(D_{g}\right)$. 
Then there exists a Riemannian metric $\widetilde{g}$ on $\widetilde{M}$ such that $D_{g}$ and $D_{\widetilde{g}}$ are $(\Lambda, \varepsilon)$ spectral close.

Here $(\Lambda, \varepsilon)$-spectral close means that the Dirac eigenvalues of $M$ and $\widetilde{M}$ in the interval $(-\Lambda, \Lambda)$ differ at most by $\varepsilon$.

Acknowledgement. We would like to thank J. Rosenberg and S. Stolz for valuable hints. Many thanks also go to the referee for helpful suggestions on how to improve the presentation of the material.

The first author was partially supported by the Research and Training Network "EDGE". The second author was supported by a Post-doctoral scholarship from the Swedish Foundation for International Cooperation in Research and Higher Education "STINT". Both authors have also been partially supported by the Research and Training Network "Geometric Analysis" funded by the European Commission.

\section{SURGERY AND THE DIRAC OPERATOR}

By a Riemannian spin manifold we mean a Riemannian manifold $(M, g)$ together with the choice of a spin structure. Hence we can form the spinor bundle $\Sigma M$ which is a Hermitian vector bundle over $M$ of rank $2^{[n / 2]}$ where $n$ is the dimension of $M$. For even $n$ the spinor bundle splits as

$$
\Sigma M=\Sigma^{+} M \oplus \Sigma^{-} M
$$

into two subbundles of equal rank. They are called half-spinor bundles of positive and negative chirality. Sections of $\Sigma M$ are spinors. The classical Dirac operator, sometimes also called Atiyah-Singer operator, is a formally self-adjoint elliptic differential operator of first order acting on the space of spinors. We denote the Dirac operator by $D$ and if we want to emphasize the dependence on the Riemannian metric we write $D_{g}$. In even dimensions it interchanges chiralities, that is with respect to splitting (1) it is of the form

$$
D_{g}=\left(\begin{array}{cc}
0 & D_{g}^{-} \\
D_{g}^{+} & 0
\end{array}\right) .
$$

For any differentiable function $f$ and spinor $\varphi$ on $M$ we have the formula

$$
D(f \varphi)=f D \varphi+\nabla f \cdot \varphi
$$

where $\nabla f$ is the gradient of $f$ and $X \cdot \varphi$ denotes Clifford multiplication of a tangent vector $X$ with a spinor $\varphi$. Another important formula concerns the square of the Dirac operator,

$$
D^{2}=\nabla^{*} \nabla+\frac{1}{4} \text { scal }
$$

where $\nabla$ is the Hermitian connection on $\Sigma M$ induced by the Levi-Civita connection and scal denotes scalar curvature. This is usually called the Lichnerowicz formula [16] but was already known to Schrödinger in the 1930's [22].

We will always consider closed manifolds $M$. Standard elliptic theory then tells us that the spectrum of $D$ is real and discrete. In particular, $D_{g}^{+}$is a Fredholm operator 
and its index is given by the celebrated Atiyah-Singer index formula

$$
\operatorname{ind}\left(D_{g}^{+}\right)=\widehat{A}(M)
$$

where $\widehat{A}(M)$ is the $\widehat{A}$-genus of $M$ constructed out of the Pontrjagin classes of $M$. There is a generalization of this index theorem to which we will return in Section 3 . See [15] for a thorough introduction to spin geometry.

Next let us recall the concept of surgery. Let $N$ be an embedded sphere of codimension $k$ in $M$ with a trivialized neighborhood, meaning there is a diffeomorphism $\Phi$ mapping $S^{n-k} \times D^{k}$ diffeomorphically onto its image in $M$ such that $S^{n-k} \times\{0\}$ is mapped onto $N$. Surgery now consists of removing $\Phi\left(S^{n-k} \times D^{k}\right)$ and gluing in $D^{n-k+1} \times$ $S^{k-1}$ along the common boundary $S^{n-k} \times S^{k-1}$. We will only perform surgeries in codimension $k \geq 3$. If $n-k \neq 1$, then $S^{n-k} \times S^{k-1}$ is simply connected (for $n-k=0$ the two components are simply connected) and carries a unique spin structure. This spin structure extends to the unique spin structure on $D^{n-k+1} \times S^{k-1}$. Thus any spin structure on $M$ induces one on the boundary of $\Phi\left(S^{n-k} \times D^{k}\right)$ which then extends uniquely to one on $\widetilde{M}$, the manifold after surgery. If $n-k=1$, then $S^{n-k} \times S^{k-1}$ has two different spin structures but only one of them extends to $D^{n-k+1} \times S^{k-1}$. In this case it will be assumed that the trivialization $\Phi$ of the neighborhood of $N$ is chosen such that it induces on the boundary the spin structure which extends. Then again we obtain a spin structure on $\widetilde{M}$.

The main theorem will roughly say that the spectrum of the Dirac operator in an arbitrarily fixed range will change only very little if one performs surgery in codimension $\geq 3$ and chooses the metric on the resulting manifold $\widetilde{M}$ carefully. To formulate this in a precise way we make the following definition.

Definition 1.1. Let $\varepsilon>0$ and $\Lambda>0$. We call two operators with discrete spectrum $(\Lambda, \varepsilon)$-spectral close if

- $\pm \Lambda$ are not eigenvalues of either operator.

- Both operators have the same total number $m$ of eigenvalues in the interval $(-\Lambda, \Lambda)$.

- If the eigenvalues in $(-\Lambda, \Lambda)$ are denoted by $\lambda_{1} \leq \cdots \leq \lambda_{m}$ and $\mu_{1} \leq \cdots \leq \mu_{m}$ respectively (each eigenvalue being repeated according to its multiplicity), then $\left|\lambda_{j}-\mu_{j}\right|<\varepsilon$ for $j=1, \ldots, m$.

Theorem 1.2. Let $(M, g)$ be a closed Riemannian manifold equipped with a spin structure. Let $N \subset M$ be an embedded sphere of codimension $k \geq 3$ and with trivialized tubular neighborhood. Let $\widetilde{M}$ be obtained from $M$ by surgery along $N$ together with the resulting spin structure. Let $\varepsilon>0$ and $\Lambda>0, \pm \Lambda \notin \operatorname{spec}\left(D_{g}\right)$. Then there exists a Riemannian metric $\widetilde{g}$ on $\widetilde{M}$ such that $D_{g}$ and $D_{\widetilde{g}}$ are $(\Lambda, \varepsilon)$ spectral close.

Remark 1.3. If $U$ is an open neighborhood of $N$ in $M$, then $\widetilde{M}$ is of the form

$$
\widetilde{M}=(M \backslash U) \cup \widetilde{U}
$$

where $\widetilde{U}$ is an open subset of $\widetilde{M}$. The proof will show that for any such $U$ the metric $\widetilde{g}$ can be chosen in such a way that it coincides with $g$ on $M \backslash U$. 
By choosing $\Lambda_{i}>0$ such that $\Lambda_{i} \rightarrow \infty$ and $\varepsilon_{i}>0$ such that $\varepsilon_{i} \rightarrow 0$ for $i \rightarrow \infty$ we obtain the following corollary.

Corollary 1.4. Let $(M, g)$ be a closed Riemannian manifold equipped with a spin structure. Let $N \subset M$ be an embedded sphere of codimension $k \geq 3$ and with trivialized tubular neighborhood. Let $\widetilde{M}$ be obtained from $M$ by surgery along $N$ together with the resulting spin structure. Then there is a sequence of Riemannian metrics $\widetilde{g}_{i}$ on $\widetilde{M}$ such that the Dirac eigenvalues of $\left(\widetilde{M}, \widetilde{g}_{i}\right)$ converge to exactly the Dirac eigenvalues of $(M, g)$.

Again we note that the metrics $\widetilde{g}_{i}$ can be chosen such that they converge to $g$ away from the sphere $N$ in the following sense: To any compact subset $K \subset M$ which does not intersect $N$ there is an isometric copy of $K$ contained in $\left(\widetilde{M}, \widetilde{g}_{i}\right)$ for $i$ sufficiently large.

The next section will be devoted to the proof of Theorem 1.2. Since the proof is somewhat involved the reader who is primarily interested in applications of the theorem may continue directly with Section 3 at a first reading.

\section{PROOF OF THE SURGERY THEOREM}

The proof of Theorem 1.2 consists of two parts. In the first part, which is quite simple, we show that to any Dirac eigenvalue of the original manifold $M$ in the range $(-\Lambda, \Lambda)$ there is a nearby eigenvalue of the manifold $\widetilde{M}$ after surgery. This is done by multiplying a corresponding eigenspinor with a suitable cut-off function and plugging the resulting spinor into the Rayleigh quotient for the Dirac operator on $\widetilde{M}$. Here the choice of metric on $\widetilde{M}$ is not so crucial. It suffices that the metric coincides with the original metric outside a sufficiently small neighborhood of the sphere along which the surgery is performed.

The second, more complicated, part consists of showing that $\widetilde{M}$ has no further Dirac eigenvalues in the interval $(-\Lambda, \Lambda)$. This requires a finer analysis of the behavior of eigenspinors. First we make the scalar curvature on $M$ very large on a neighborhood $U$ of the surgery sphere by a $C^{1}$-small deformation of the metric (Section 2.1). This property persists under surgery of codimension $\geq 3$. Here we use the construction of Gromov and Lawson which shows that the class of manifolds admitting metrics of positive scalar curvature is closed under surgery of codimension $\geq 3$. Hence scalar curvature is very large in the corresponding open subset $\widetilde{U}$ of $\widetilde{M}$. This implies that most of the $L^{2}$-norm of an eigenspinor is supported in the complement $\widetilde{M} \backslash \widetilde{U}=$ $M \backslash U$ (Section 2.2). Then we do a finer analysis of the distribution of the $L^{2}$-norm on certain annular regions (Section 2.3). This finally allows us to multiply an eigenspinor of $\widetilde{M}$ with a cut-off function whose gradient is supported in such an annular region where the eigenspinor carries only very little $L^{2}$-norm. Therefore the bad error term in the Rayleigh quotient coming from the gradient of the cut-off function is under good control. It follows that to each eigenvalue of $\widetilde{M}$ there corresponds one of $M$ and we are done. 
It should be noted that the proof is not at all symmetric in $M$ and $\widetilde{M}$ because the metric on $\widetilde{M}$ is not given beforehand and therefore various geometric quantities are not under a-priori control. To make the second part work we must choose the metric on $\widetilde{M}$ very carefully.

2.1. Increasing scalar curvature along submanifolds. In this subsection we show that the scalar curvature can be made large near a submanifold by a $C^{1}$-small deformation of the metric (which does not change the Dirac eigenvalues much). Then Lemma 2.2 of the next subsection will show that only little $L^{2}$-norm of eigenspinors is contained in the problematical region near the sphere along which we will perform surgery.

Proposition 2.1. Let $(M, g)$ be an n-dimensional Riemannian manifold, let $N \subset M$ be a compact submanifold of positive codimension. Let $U$ be a compact neighborhood of $N$ in $M$. Then there exists a sequence of smooth Riemannian metrics $g_{j}$ on $M$ such that

- $g_{j}$ is conformally equivalent to $g$,

- $g_{j}=g$ on $M \backslash U$,

- $g_{j} \rightarrow g$ in the $C^{1}$-topology as $j \rightarrow \infty$,

- $\min _{N} \operatorname{scal}_{g_{j}} \rightarrow \infty$ as $j \rightarrow \infty$,

- there exists a constant $S_{0} \in \mathbb{R}$ such that on $U$ and for all $j$

$$
\operatorname{scal}_{g_{j}} \geq S_{0} \text {. }
$$

Proof. The scalar curvature of a conformally equivalent metric $\widehat{g}=e^{2 u} g$ is given by

$$
\operatorname{scal}_{\widehat{g}}=e^{-2 u}\left(\operatorname{scal}_{g}+2(n-1) \Delta u-(n-2)(n-1)|d u|^{2}\right) \text {, }
$$

see [4. Thm. 1.159]. Hence it is sufficient to find a sequence of smooth functions $u_{j}$ on $M$ such that

- $u_{j}=0$ on $M \backslash U$,

- $u_{j} \rightarrow 0$ uniformly,

- $d u_{j} \rightarrow 0$ uniformly,

- $\Delta u_{j}$ uniformly bounded from below and

- $\min _{N} \Delta u_{j} \rightarrow \infty$ as $j \rightarrow \infty$.

To construct such functions we first assume that the normal bundle of $N$ in $M$ contains a trivial line bundle, that is we assume there is a smooth unit normal field $\nu$ on $N$. Choose a relatively compact neighborhood $U_{1}$ of $N$ in $U$ sufficiently small so that the Riemannian exponential map maps a neighborhood $\widehat{U}$ of the zero section in the normal bundle diffeomorphically onto $U_{1}$,

$$
\left.\exp \right|_{\widehat{U}}: \widehat{U} \stackrel{\approx}{\longrightarrow} U_{1} \subset \subset U \subset M .
$$

We define a smooth function $\rho$ by $\rho(x)=g\left(\left(\left.\exp \right|_{\widehat{U}}\right)^{-1}(x), \nu\right)$ on $U_{1}$. On $M \backslash U$ we define $\rho$ to be 1 , and we extend $\rho$ in some way to $U \backslash U_{1}$ so that we obtain a smooth function defined on all of $M$ with the following properties. 
- $\rho \equiv 1$ on $M \backslash U$,

- $\rho \equiv 0$ on $N$,

- $\operatorname{grad} \rho=\nu$ on $N$, in particular $|d \rho| \equiv 1$ on $N$,

- $|d \rho| \leq C_{1}$ on $M$,

- $|\Delta \rho| \leq C_{2}$ on $M$.

Next, we pick continuous functions $\widehat{\varphi}_{j}: \mathbb{R} \rightarrow \mathbb{R}$, smooth on $\mathbb{R} \backslash\{0\}$ such that

- $\widehat{\varphi}_{j}(0)=\frac{1}{j}$

- $0 \leq \widehat{\varphi}_{j} \leq \frac{1}{j}$ on $\mathbb{R}$,

- $\widehat{\varphi}_{j} \equiv 0$ on $\mathbb{R} \backslash(-1,1)$,

- $\widehat{\varphi}_{j}^{\prime}=\frac{2}{j}$ on $\left[-\frac{1}{4}, 0\right), \widehat{\varphi}_{j}^{\prime}=-\frac{2}{j}$ on $\left(0, \frac{1}{4}\right]$,

- $\left|\widehat{\varphi}_{j}^{\prime}\right| \leq \frac{2}{j}$ on $\mathbb{R} \backslash\{0\}$,

- $\left|\widehat{\varphi}_{j}^{\prime \prime}\right| \leq \frac{C_{3}}{j}$ on $\mathbb{R} \backslash\{0\}$.

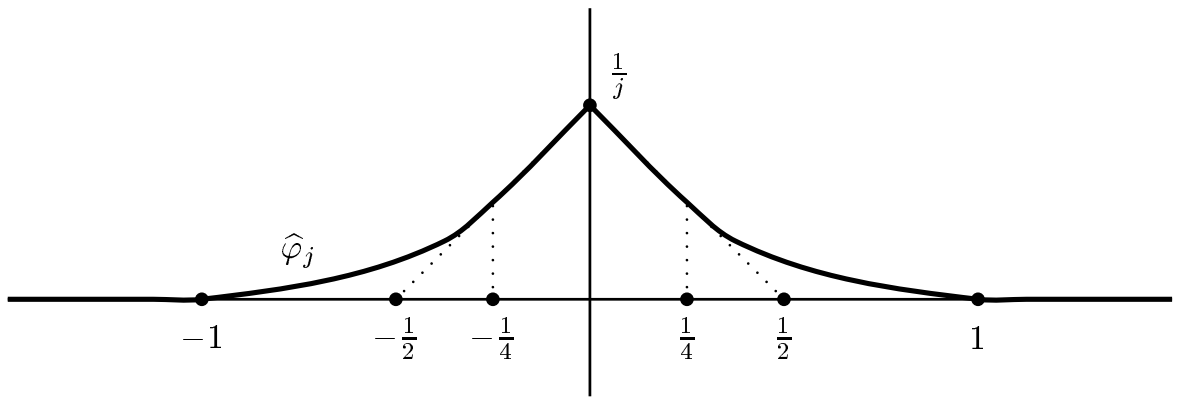

Fig. 1

Note that $\widehat{\varphi}_{j}$ can simply be chosen as $\frac{1}{j} \widehat{\varphi}_{1}$. We smooth out $\widehat{\varphi}_{j}$ in a neighborhood of 0 with size of order $\frac{1}{j}$ and obtain smooth functions $\varphi_{j}: \mathbb{R} \rightarrow \mathbb{R}$ with :

- $0 \leq \varphi_{j} \leq \frac{1}{j}$ on $\mathbb{R}$,

- $\left|\varphi_{j}^{\prime}\right| \leq \frac{2}{j}$ on $\mathbb{R}$,

- $\varphi_{j}^{\prime \prime} \leq \frac{C_{3}}{j}$ on $\mathbb{R}$,

- $\varphi_{j} \equiv 0$ on $\mathbb{R} \backslash(-1,1)$,

- $\varphi_{j}^{\prime \prime}(0) \leq-j$. 


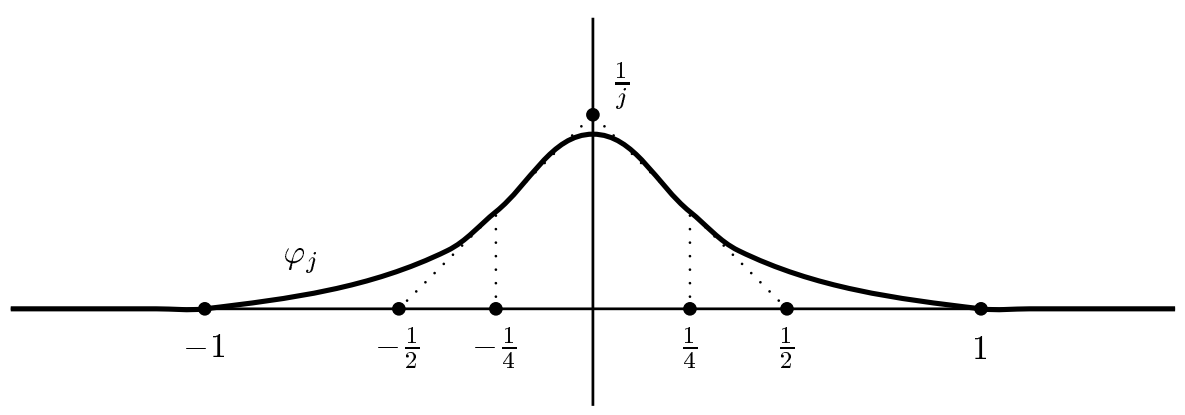

Fig. 2

One checks easily that $u_{j}:=\varphi_{j} \circ \rho$ has all the required properties (using the formula $\left.\Delta u_{j}=\left(\varphi_{j}^{\prime} \circ \rho\right) \Delta \rho-\left(\varphi_{j}^{\prime \prime} \circ \rho\right)|d \rho|^{2}\right)$.

It remains to handle the case when the normal bundle of $N$ in $M$ has no trivial onedimensional sub-bundle. Locally it always has. Hence we can cover $N$ by open sets $V_{k}, k=1, \ldots, \ell$, and find smooth functions $\rho_{k}: M \rightarrow \mathbb{R}$ having all the properties of the previous $\rho$ on $V_{k}$ instead of $N$. Let $\chi_{1}, \ldots, \chi_{\ell+1}$ be a partition of unity on $M$ subordinate to the open covering $\exp \left(\pi^{-1}\left(V_{1}\right) \cap \widetilde{U}\right), \ldots, \exp \left(\pi^{-1}\left(V_{\ell}\right) \cap \widetilde{U}\right), M \backslash$ $N$. Then $\left.\chi_{1}\right|_{N}, \ldots,\left.\chi_{\ell}\right|_{N}$ form a partition of unity on $N$ subordinate to the covering $V_{1}, \ldots, V_{\ell}$. Again, it is easy to check that

$$
u_{j}:=\sum_{k=1}^{\ell+1} \chi_{k} \cdot\left(\varphi_{j} \circ \rho_{k}\right)
$$

has the required properties.

2.2. Distribution of $\mathbf{L}^{\mathbf{2}}$-norm of eigenspinors and scalar curvature. Next we show that eigenspinors try to avoid large scalar curvature, that is, most of their $L^{2}$-norm is supported in the region of the manifold where the scalar curvature is small.

Lemma 2.2. Let $M$ be a closed Riemannian spin manifold. Let $\Lambda, S_{0}, S_{1} \in \mathbb{R}, S_{0}<$ $S_{1}$. Suppose the scalar curvature of $M$ satisfies scal $\geq S_{0}$. Put

$$
M_{+}:=\left\{x \in M \mid \operatorname{scal}(x) \geq S_{1}\right\} .
$$

Then for any spinor satisfying

$$
\|D \psi\|_{L^{2}(M)}^{2} \leq \Lambda^{2}\|\psi\|_{L^{2}(M)}^{2}
$$

the following inequality holds:

$$
\int_{M_{+}}|\psi|^{2} \mathrm{dvol} \leq \frac{4 \Lambda^{2}-S_{0}}{S_{1}-S_{0}} \int_{M}|\psi|^{2} \mathrm{dvol} .
$$


Proof. Using the Lichnerowicz formula $D^{2}=\nabla^{*} \nabla+\frac{1}{4}$ scal we obtain

$$
\begin{aligned}
0 & \leq \int_{M}|\nabla \psi|^{2} \mathrm{dvol}=\int_{M}\left\langle\nabla^{*} \nabla \psi, \psi\right\rangle \mathrm{dvol} \\
& =\int_{M}\left\langle D^{2} \psi, \psi\right\rangle \mathrm{dvol}-\frac{1}{4} \int_{M} \operatorname{scal}|\psi|^{2} \mathrm{dvol} \\
& \leq \Lambda^{2} \int_{M}|\psi|^{2} \mathrm{dvol}-\frac{S_{0}}{4} \int_{M \backslash M_{+}}|\psi|^{2} \mathrm{dvol}-\frac{S_{1}}{4} \int_{M_{+}}|\psi|^{2} \mathrm{dvol} .
\end{aligned}
$$

Hence

$$
\left(\frac{S_{1}}{4}-\Lambda^{2}\right) \int_{M_{+}}|\psi|^{2} \mathrm{dvol} \leq\left(\Lambda^{2}-\frac{S_{0}}{4}\right) \int_{M \backslash M_{+}}|\psi|^{2} \mathrm{dvol}
$$

and therefore

$$
\left(\frac{S_{1}}{4}-\frac{S_{0}}{4}\right) \int_{M_{+}}|\psi|^{2} \mathrm{dvol} \leq\left(\Lambda^{2}-\frac{S_{0}}{4}\right) \int_{M}|\psi|^{2} \mathrm{dvol}
$$

which is the claim of the lemma.

Remark 2.3. The lemma can be slightly improved to

$$
\int_{M_{+}}|\psi|^{2} \mathrm{dvol} \leq \frac{4 \frac{n-1}{n} \Lambda^{2}-S_{0}}{S_{1}-S_{0}} \int_{M}|\psi|^{2} \mathrm{dvol}
$$

with essentially the same proof. One combines the Lichnerowicz formula with the identity

$$
|\nabla \psi|^{2}=|P \psi|^{2}+\frac{1}{n}|D \psi|^{2}
$$

where $P$ is the twistor operator. Estimating $\int_{M}|P \psi|^{2}$ dvol (instead of $\int_{M}|\nabla \psi|^{2}$ dvol) by 0 one looses a little less. Note that the estimate is nontrivial only for

$$
\Lambda^{2} \leq \frac{n}{n-1} \frac{S_{1}}{4}
$$

because otherwise the coefficient on the right hand side of (2) is greater than 1 . The estimate also shows that if $\Lambda^{2} \leq \frac{n}{n-1} \frac{S_{0}}{4}$, then $\psi=0$. This way we recover Friedrich's eigenvalue estimate [8]:

$$
\lambda^{2} \geq \frac{n}{n-1} \frac{S_{0}}{4} .
$$

In our application however $S_{0}$ will typically be negative.

2.3. $\mathbf{L}^{2}$-norm on annular regions. In the following we will look at the "distance spheres" of a compact submanifold $N$ of a Riemannian manifold $M$ given by

$$
S_{N}(r):=\{x \in M \mid \operatorname{dist}(x, N)=r\}
$$

for $r>0$. For $0 \leq R_{1}<R_{2}$ we define the annular region

$$
A_{N}\left(R_{1}, R_{2}\right):=\left\{x \in M \mid R_{1} \leq \operatorname{dist}(x, N) \leq R_{2}\right\}=\bigcup_{R_{1} \leq r \leq R_{2}} S_{N}(r) .
$$

Let $\nu$ denote the unit normal vector field of $S_{N}(r)$ pointing away from $N$, let $W=$ $-\nabla \nu$ denote the Weingarten map (or shape operator) and let $H=\frac{1}{n-1} \operatorname{tr}(W)$ be the mean curvature of $S_{N}(r)$ with respect to $\nu$. The following lemma tells us that 
under a suitable condition only little $L^{2}$-norm of a spinor is contained in the annulus $A_{N}(r, 2 r)$ compared to the bigger annulus $A_{N}\left(r,(2 r)^{1 / 11}\right)$ when $r$ is small.

Lemma 2.4. Let $M$ be an n-dimensional Riemannian spin manifold and let $N \subset M$ be a compact submanifold of codimension $k \geq 3$. Then there exists $0<R<1$ depending on $M$ and $N$ such that for any $0<r \leq \frac{1}{2} R^{11}$ and any smooth spinor $\varphi$ defined on $A_{N}\left(r,(2 r)^{1 / 11}\right)$ the following estimate holds

$$
\frac{\|\varphi\|_{L^{2}\left(A_{N}(r, 2 r)\right)}^{2}}{\|\varphi\|_{L^{2}\left(A_{N}\left(r,(2 r)^{1 / 11}\right)\right)}^{2}} \leq 10 r^{5 / 2}
$$

provided

$$
\operatorname{Re} \int_{S_{N}(\rho)}\left\langle\nabla_{\nu} \varphi, \varphi\right\rangle d A \geq 0
$$

holds for all $\rho \in\left[r,(2 r)^{1 / 11}\right]$.

Proof. From

$$
\frac{d}{d \rho} d A=-(n-1) H d A
$$

and

$$
\frac{d}{d \rho}|\varphi|^{2}=2 \operatorname{Re}\left\langle\nabla_{\nu} \varphi, \varphi\right\rangle
$$

we conclude

$$
\begin{aligned}
\frac{d}{d \rho} \int_{S_{N}(\rho)}|\varphi|^{2} d A & =\operatorname{Re} \int_{S_{N}(\rho)}\left\{2\left\langle\nabla_{\nu} \varphi, \varphi\right\rangle-(n-1) H|\varphi|^{2}\right\} d A \\
& \geq-(n-1) \int_{S_{N}(\rho)} H|\varphi|^{2} d A .
\end{aligned}
$$

For $p \in N$ and $\nu$ a unit normal vector to $N$ at $p$ let $P_{\nu}$ denote the orthogonal projection

$$
P_{\nu}: \nu^{\perp} \longrightarrow \nu^{\perp} \cap T_{p} N^{\perp}
$$

In particular, we have the orthogonal decomposition

$$
T_{p} M=T_{p} N \oplus \mathbb{R} \nu \oplus \operatorname{im}\left(P_{\nu}\right) .
$$

Let $P_{\nu, \rho}$ denote the parallel translate of $P_{\nu}$ along $c(\rho)=\exp (\rho \nu)$. Then $W$ is of the form

$$
W(c(\rho))=-\frac{1}{\rho} P_{\nu, \rho}+C(\rho),
$$

where $C(\rho)$ extends smoothly to $\rho=0$, compare [7]. Since the rank of $P_{\nu}$ is equal to $k-1$ we conclude that

$$
(n-1) H=-\frac{k-1}{\rho}+\mathrm{O}_{M, N}(1) .
$$

For $0<\rho \leq R$, where $R$ is sufficiently small, we have

$$
\left|\mathrm{O}_{M, N}(1)\right| \leq \frac{1}{4 \rho}
$$


and therefore

$$
(n-1) H \leq-\frac{k-5 / 4}{\rho} .
$$

Plugging this into (3) we get

$$
\frac{d}{d \rho} \int_{S_{N}(\rho)}|\varphi|^{2} d A \geq \frac{k-5 / 4}{\rho} \int_{S_{N}(\rho)}|\varphi|^{2} d A \geq \frac{7 / 4}{\rho} \int_{S_{N}(\rho)}|\varphi|^{2} d A .
$$

since $k \geq 3$. Therefore

$$
\frac{d}{d \rho} \ln \int_{S_{N}(\rho)}|\varphi|^{2} d A \geq \frac{7 / 4}{\rho}
$$

and hence for $r_{1} \leq r_{2}$

$$
\begin{aligned}
\ln \left(\frac{\int_{S_{N}\left(r_{2}\right)}|\varphi|^{2} d A}{\int_{S_{N}\left(r_{1}\right)}|\varphi|^{2} d A}\right) & =\ln \left(\int_{S_{N}\left(r_{2}\right)}|\varphi|^{2} d A\right)-\ln \left(\int_{S_{N}\left(r_{1}\right)}|\varphi|^{2} d A\right) \\
& \geq \frac{7}{4} \int_{r_{1}}^{r_{2}} \frac{d \rho}{\rho} \\
& =\frac{7}{4} \ln \left(\frac{r_{2}}{r_{1}}\right) .
\end{aligned}
$$

Exponentiation yields

$$
\frac{\int_{S_{N}\left(r_{2}\right)}|\varphi|^{2} d A}{\int_{S_{N}\left(r_{1}\right)}|\varphi|^{2} d A} \geq\left(\frac{r_{2}}{r_{1}}\right)^{7 / 4} .
$$

From (3) and $H<0$ it follows that the function $\rho \mapsto \int_{S_{N}(\rho)}|\varphi|^{2} d A$ is monotonically increasing, so we have on the one hand

$$
\|\varphi\|_{L^{2}\left(A_{N}(r, 2 r)\right)}^{2}=\int_{r}^{2 r} \int_{S_{N}(\rho)}|\varphi|^{2} d A d \rho \leq r \int_{S_{N}(2 r)}|\varphi|^{2} d A .
$$

On the other hand by (\#) with $r_{1}=2 r$ and $r_{2}=\rho$,

$$
\begin{aligned}
\|\varphi\|_{L^{2}\left(A_{N}\left(2 r,(2 r)^{1 / 11}\right)\right)}^{2} & =\int_{2 r}^{(2 r)^{1 / 11}} \int_{S_{N}(\rho)}|\varphi|^{2} d A d \rho \\
& \geq \int_{S_{N}(2 r)}|\varphi|^{2} d A \int_{2 r}^{(2 r)^{1 / 11}}\left(\frac{\rho}{2 r}\right)^{7 / 4} d \rho \\
& =\int_{S_{N}(2 r)}|\varphi|^{2} d A \cdot \frac{8 r}{11}\left[\left(\frac{1}{2 r}\right)^{5 / 2}-1\right] .
\end{aligned}
$$

Combining (5) and (6) we obtain

$$
\begin{aligned}
\frac{\|\varphi\|_{L^{2}\left(A_{N}\left(r,(2 r)^{1 / 11}\right)\right)}^{2}}{\|\varphi\|_{L^{2}\left(A_{N}(r, 2 r)\right)}^{2}} & =1+\frac{\|\varphi\|_{L^{2}\left(A_{N}\left(2 r,(2 r)^{1 / 11}\right)\right)}^{2}}{\|\varphi\|_{L^{2}\left(A_{N}(r, 2 r)\right)}^{2}} \\
& \geq 1+\frac{8}{11}\left[\left(\frac{1}{2 r}\right)^{5 / 2}-1\right] \\
& >\frac{1}{10}\left(\frac{1}{r}\right)^{5 / 2},
\end{aligned}
$$


and the lemma follows.

2.4. Proof of Theorem 1.2. By decreasing $\varepsilon$ if necessary we may assume that $(\Lambda-$ $\varepsilon, \Lambda+\varepsilon)$ and $(-\Lambda-\varepsilon,-\Lambda+\varepsilon)$ contain no eigenvalues of $D_{g}$ and that any two distinct eigenvalues in $(-\Lambda, \Lambda)$ are at distance at least $2 \varepsilon$. Write $\lambda_{1} \leq \lambda_{2} \leq \cdots \leq \lambda_{m}$ for the eigenvalues of $D_{g}$ in $(-\Lambda, \Lambda)$, each eigenvalue being repeated according to its multiplicity. Denote the distance tube of radius $r$ about $N$ by $U_{N}(r)$, that is

$$
U_{N}(r)=\{x \in M \mid \operatorname{dist}(x, N)<r\} .
$$

We will first show that there exists $R>0$ such that the Dirac operator $D_{\widetilde{g}}$ on any closed Riemannian spin manifold $(\widetilde{M}, \widetilde{g})$ containing an isometric copy of $M \backslash U_{N}(r)$ (together with its spin structure) has eigenvalues $\mu_{1} \leq \mu_{2} \leq \cdots \leq \mu_{m}$ with $\left|\lambda_{i}-\mu_{i}\right|<$ $\varepsilon$ provided $r<R$. It may however have further eigenvalues in the interval $(-\Lambda, \Lambda)$. This will be excluded later under additional assumptions.

To prove the existence of the eigenvalues $\mu_{i}$ let $\chi_{r}: M \rightarrow \mathbb{R}$ be a smooth cut-off function with the following properties

- $0 \leq \chi_{r} \leq 1$ on $M$,

- $\chi_{r} \equiv 0$ on $U_{N}(r)$,

- $\chi_{r} \equiv 1$ on $M \backslash U_{N}(2 r)$,

- $\left|\nabla \chi_{r}\right| \leq \frac{2}{r}$ on $M$.

Since all norms on a finite dimensional vector space are equivalent there exists a constant $C_{1}>0$ such that

$$
\|\varphi\|_{L^{\infty}(M)} \leq C_{1}\|\varphi\|_{L^{2}(M)}
$$

for all eigenspinors $\varphi$ of $D_{g}$ with eigenvalue in $(-\Lambda, \Lambda)$. Moreover, there is a constant $C_{2}>0$ such that

$$
\operatorname{vol}\left(U_{N}(2 r)\right) \leq C_{2} r^{k} .
$$

Let $\varphi_{i}$ be an eigenspinor on $M$ for the eigenvalue $\lambda_{i}$. Since $\chi_{r} \varphi_{i}$ has its support in $M \backslash U_{N}(r)$ it can also be regarded as a spinor on $\widetilde{M}$. We plug it into the Rayleigh quotient of $D_{\widetilde{g}}-\lambda_{i}$ :

$$
\begin{aligned}
\frac{\left\|\left(D_{\widetilde{g}}-\lambda_{i}\right)\left(\chi_{r} \varphi_{i}\right)\right\|_{L^{2}(\widetilde{M})}^{2}}{\left\|\chi_{r} \varphi_{i}\right\|_{L^{2}(\widetilde{M})}^{2}} & =\frac{\left\|\nabla \chi_{r} \cdot \varphi_{i}\right\|_{L^{2}\left(U_{N}(2 r)\right)}^{2}}{\left\|\chi_{r} \varphi_{i}\right\|_{L^{2}(M)}^{2}} \\
& \leq \frac{\frac{4}{r^{2}}\left\|\varphi_{i}\right\|_{L^{2}\left(U_{N}(2 r)\right)}^{2}}{\left\|\varphi_{i}\right\|_{L^{2}(M)}^{2}-\left\|\varphi_{i}\right\|_{L^{2}\left(U_{N}(2 r)\right)}^{2}}
\end{aligned}
$$

From

$$
\begin{aligned}
\left\|\varphi_{i}\right\|_{L^{2}\left(U_{N}(2 r)\right)}^{2} & \leq\left\|\varphi_{i}\right\|_{L^{\infty}\left(U_{N}(2 r)\right)}^{2} \operatorname{vol}\left(U_{N}(2 r)\right) \\
& \leq C_{1}^{2}\left\|\varphi_{i}\right\|_{L^{2}(M)}^{2} C_{2} r^{k}
\end{aligned}
$$


we conclude

$$
\begin{aligned}
\frac{\left\|\left(D_{\widetilde{g}}-\lambda_{i}\right)\left(\chi_{r} \varphi_{i}\right)\right\|_{L^{2}(\widetilde{M})}^{2}}{\left\|\chi_{r} \varphi_{i}\right\|_{L^{2}(\widetilde{M})}^{2}} & \leq \frac{\frac{4}{r^{2}} C_{1}^{2} C_{2} r^{k}}{1-C_{1}^{2} C_{2} r^{k}} \\
& =\frac{4 C_{1}^{2} C_{2} r^{k-2}}{1-C_{1}^{2} C_{2} r^{k}} \\
& <\varepsilon^{2}
\end{aligned}
$$

for $r$ sufficiently small because $k \geq 3$. Therefore the operator $D_{\widetilde{g}}-\lambda_{i}$ has an eigenvalue in the interval $(-\varepsilon, \varepsilon)$, hence $D_{\widetilde{g}}$ has an eigenvalue $\mu_{i}$ in $\left(\lambda_{i}-\varepsilon, \lambda_{i}+\varepsilon\right)$. If $\lambda_{i}$ has multiplicity $\ell$ this yields $\ell$ eigenvalues of $D_{\widetilde{g}}$ in $\left(\lambda_{i}-\varepsilon, \lambda_{i}+\varepsilon\right)$.

It remains to be shown that in the situation of the theorem $\widetilde{g}$ can be chosen so that the operator $D_{\widetilde{g}}$ has no more than $m$ eigenvalues in $(-\Lambda, \Lambda)$. According to Proposition 2.1 there is a metric on $M$ arbitrarily close to $g$ in the $C^{1}$-topology such that with respect to this metric

- $\quad$ scal $\geq S_{0}$ on all of $M$,

- scal $\geq 2 S_{1}$ on a neighborhood $U_{0}$ of $N$ with $S_{1}$ arbitrarily large.

Since the eigenvalues of $D_{g}$ depend continuously on the Riemannian metric with respect to the $C^{1}$-topology (see for example [3, Prop. 7.1]) we may assume without loss of generality that the scalar curvature of $g$ itself has these properties with $S_{1}$ so large that

$$
\frac{S_{1}-4 \Lambda^{2}}{S_{1}-S_{0}} \geq\left(\frac{\Lambda+\varepsilon / 2}{\Lambda+\varepsilon}\right)^{2}
$$

Now choose $r>0$ so small that

- $r<\frac{\varepsilon^{4}}{2^{8} \cdot 100 \cdot(m+1)^{4}}$,

- $U_{N}\left((2 r)^{1 / 11}\right) \subset U_{0}$,

- $(2 r)^{1 / 11}$ is no larger than the $R$ in Lemma 2.4.

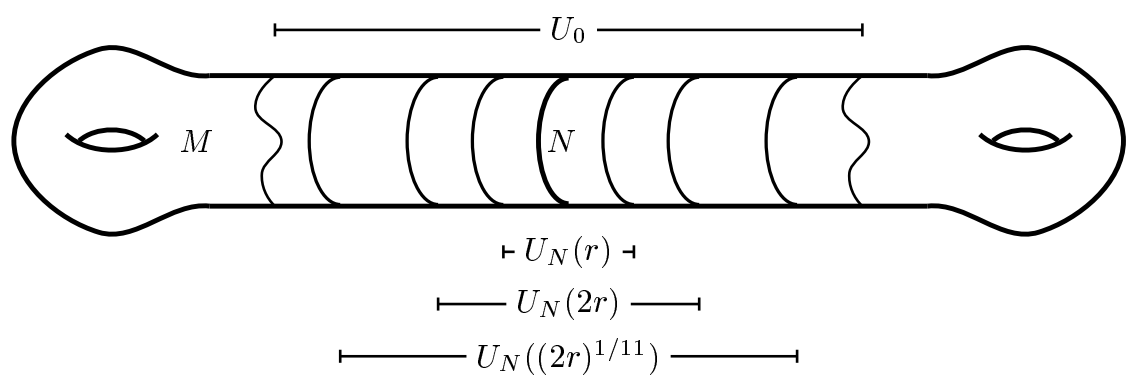

Fig. 3 
We perform the surgery along $N$ in the neighborhood $U=U_{N}(r)$. Hence $\widetilde{M}$ is of the form $\widetilde{M}=\left(M \backslash U_{N}(r)\right) \cup \widetilde{U}$. Surgery in codimension $\geq 3$ does not decrease scalar curvature too much if the metric $\widetilde{g}$ on $\widetilde{M}$ is chosen properly, see 9 , Proof of Theorem A] and [21, Proof of Theorem 3.1]. We may assume

- $\operatorname{scal}_{\widetilde{g}} \geq S_{0}$ on all of $\widetilde{M}$,

- $\operatorname{scal}_{\tilde{g}} \geq S_{1}$ on $\widetilde{U}$.

We will show that with these choices of $r$ and $\widetilde{g}$ the Dirac operator of $\widetilde{M}$ can have no more than $m$ eigenvalues in $(-\Lambda, \Lambda)$. Assume the contrary, that is, assume there is an $(m+1)$-dimensional space $\widetilde{\mathcal{H}}$ of spinors on $\widetilde{M}$ spanned by eigenspinors with eigenvalues in $(-\Lambda, \Lambda)$. The function $\chi_{r}$ can also be considered as a cut-off function on $\widetilde{M}$ since its support is contained in $M \backslash U_{N}(r)$ which is contained in $\widetilde{M}$. For every spinor $\varphi$ on $\widetilde{M}$ the spinor $\chi_{r} \varphi$ can be considered a spinor also on $M$. The space

$$
\mathcal{H}:=\left\{\chi_{r} \varphi \mid \varphi \in \widetilde{\mathcal{H}}\right\}
$$

has the same dimension $m+1$ as $\widetilde{\mathcal{H}}$ by the unique-continuation property of the Dirac operator (see for example [5]) and we consider it as a space of spinors on $M$. We will show that

$$
\frac{\left\|D_{g} \psi\right\|_{L^{2}(M)}^{2}}{\|\psi\|_{L^{2}(M)}^{2}}<(\Lambda+\varepsilon)^{2}
$$

for all nonzero $\psi \in \mathcal{H}$. Then $D_{g}$ must have at least $m+1$ eigenvalues in $(-\Lambda-$ $\varepsilon, \Lambda+\varepsilon)$ which is a contradiction.

Let $\psi:=\chi_{r} \varphi \in \mathcal{H}$. We write $\varphi=\varphi_{1}+\cdots+\varphi_{m+1}$ with $D_{\widetilde{g}}$-eigenspinors $\varphi_{j}$ (some possibly zero) for the eigenvalues $\mu_{j} \in(-\Lambda, \Lambda)$. By the assumption on the scalar curvature of $\widetilde{g}$ (recall that scal $\geq S_{1}$ on $\widetilde{U}$ and scal $\geq 2 S_{1}$ on $A_{N}(r, 2 r)$ ) and by Lemma 2.2 applied to $\widetilde{M}$ we have

$$
\|\varphi\|_{L^{2}\left(\widetilde{U} \cup A_{N}(r, 2 r)\right)}^{2} \leq \frac{4 \Lambda^{2}-S_{0}}{S_{1}-S_{0}}\|\varphi\|_{L^{2}(\widetilde{M})}^{2} .
$$

Thus

$$
\begin{aligned}
\|\psi\|_{L^{2}(M)}^{2} & =\left\|\chi_{r} \varphi\right\|_{L^{2}(M)}^{2} \geq\|\varphi\|_{L^{2}\left(M \backslash U_{N}(2 r)\right)}^{2} \\
& \geq\left[1-\frac{4 \Lambda^{2}-S_{0}}{S_{1}-S_{0}}\right] \cdot\|\varphi\|_{L^{2}(\widetilde{M})}^{2} \\
& =\frac{S_{1}-4 \Lambda^{2}}{S_{1}-S_{0}}\|\varphi\|_{L^{2}(\widetilde{M})}^{2} \\
& \geq\left(\frac{\Lambda+\frac{\varepsilon}{2}}{\Lambda+\varepsilon}\right)^{2}\|\varphi\|_{L^{2}(\widetilde{M})}^{2}
\end{aligned}
$$


Secondly,

$$
\begin{aligned}
\left\|D_{g} \psi\right\|_{L^{2}(M)} & =\left\|D_{\widetilde{g}}\left(\chi_{r} \varphi\right)\right\|_{L^{2}(\widetilde{M})} \\
& =\left\|\nabla \chi_{r} \cdot \varphi+\chi_{r} D_{\widetilde{g}} \varphi\right\|_{L^{2}(\widetilde{M})} \\
& \leq\left\|\nabla \chi_{r} \cdot \varphi\right\|_{L^{2}(\widetilde{M})}+\left\|D_{\widetilde{g}} \varphi\right\|_{L^{2}(\widetilde{M})} \\
& \leq \frac{2}{r}\|\varphi\|_{L^{2}\left(A_{N}(r, 2 r)\right)}+\Lambda\|\varphi\|_{L^{2}(\widetilde{M})} .
\end{aligned}
$$

Now we justify that we can apply Lemma 2.4 to the eigenspinors $\varphi_{j}$. Fix $\rho \in\left[r,(2 r)^{1 / 11}\right]$. We have to show

$$
\operatorname{Re} \int_{S_{N}(\rho)}\left\langle\nabla_{\nu} \varphi_{j}, \varphi_{j}\right\rangle d A \geq 0 .
$$

Set $\widehat{M}:=\widetilde{U} \cup A_{N}(r, \rho)$. Then $\widehat{M} \subset \widetilde{M}$ is a compact manifold with boundary $\partial \widehat{M}=$ $S_{N}(\rho)$ and scal $\geq S_{1}$ on $\widehat{M}$.

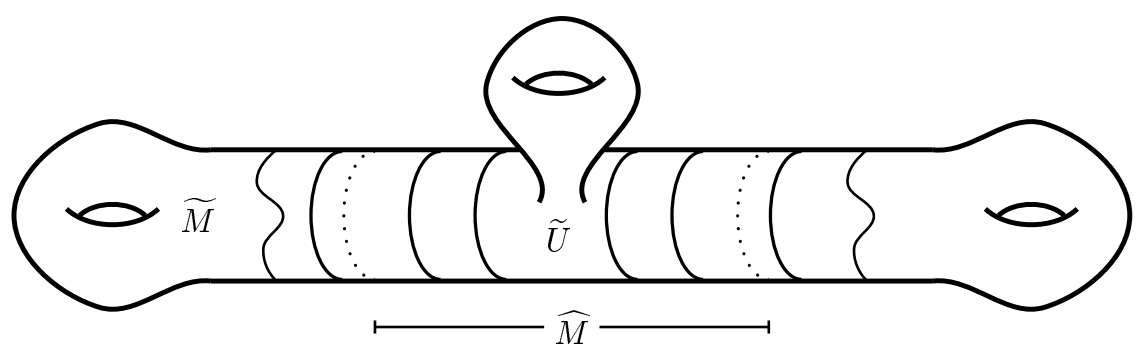

Fig. 4

The Lichnerowicz formula and a partial integration yield

$$
\begin{aligned}
\mu_{j}^{2}\left\|\varphi_{j}\right\|_{L^{2}(\widehat{M})}^{2} & =\int_{\widehat{M}}\left\langle D^{2} \varphi_{j}, \varphi_{j}\right\rangle \mathrm{dvol} \\
& =\int_{\widehat{M}}\left\langle\nabla^{*} \nabla \varphi_{j}, \varphi_{j}\right\rangle \mathrm{dvol}+\frac{1}{4} \int_{\widehat{M}} \mathrm{scal}\left|\varphi_{j}\right|^{2} \mathrm{dvol} \\
& \geq\left\|\nabla \varphi_{j}\right\|_{L^{2}(\widehat{M})}^{2}-\int_{\partial \widehat{M}}\left\langle\nabla_{\nu} \varphi_{j}, \varphi_{j}\right\rangle d A+\frac{S_{1}}{4}\left\|\varphi_{j}\right\|_{L^{2}(\widehat{M})}^{2} .
\end{aligned}
$$

Hence

$$
\int_{S_{N}(\rho)}\left\langle\nabla_{\nu} \varphi_{j}, \varphi_{j}\right\rangle d A \geq\left(\frac{S_{1}}{4}-\Lambda^{2}\right)\left\|\varphi_{j}\right\|_{L^{2}(\widehat{M})}^{2} \geq 0 .
$$

Thus Lemma 2.4 can be applied and yields

$$
\begin{aligned}
\left\|\varphi_{j}\right\|_{L^{2}\left(A_{N}(r, 2 r)\right)}^{2} & \leq 10 r^{5 / 2}\left\|\varphi_{j}\right\|_{L^{2}\left(A_{N}\left(r,(2 r)^{1 / 11}\right)\right)}^{2} \\
& \leq 10 r^{5 / 2}\left\|\varphi_{j}\right\|_{L^{2}(\widetilde{M})}^{2} \\
& \leq 10 r^{5 / 2}\|\varphi\|_{L^{2}(\widetilde{M})}^{2} .
\end{aligned}
$$


Thus

$$
\begin{aligned}
\frac{2}{r}\|\varphi\|_{L^{2}\left(A_{N}(r, 2 r)\right)} & \leq \frac{2}{r}\left\{\left\|\varphi_{1}\right\|_{L^{2}\left(A_{N}(r, 2 r)\right)}+\cdots+\left\|\varphi_{m+1}\right\|_{L^{2}\left(A_{N}(r, 2 r)\right)}\right\} \\
& \leq \frac{2}{r}(m+1) \sqrt{10 \cdot r^{5 / 2}}\|\varphi\|_{L^{2}(\widetilde{M})} \\
& =2 \sqrt{10}(m+1) r^{1 / 4}\|\varphi\|_{L^{2}(\widetilde{M})} \\
& <\frac{\varepsilon}{2}\|\varphi\|_{L^{2}(\widetilde{M})} .
\end{aligned}
$$

Plugging this into $(\mathbb{7})$ gives

$$
\left\|D_{g} \psi\right\|_{L^{2}(M)}<\left(\Lambda+\frac{\varepsilon}{2}\right)\|\varphi\|_{L^{2}(\widetilde{M})}
$$

and therefore

$$
\frac{\left\|D_{g} \psi\right\|_{L^{2}(M)}^{2}}{\|\psi\|_{L^{2}(M)}^{2}}<\frac{\left(\Lambda+\frac{\varepsilon}{2}\right)^{2}\|\varphi\|_{L^{2}(\widetilde{M})}^{2}}{\left(\frac{\Lambda+\frac{\varepsilon}{2}}{\Lambda+\varepsilon}\right)^{2}\|\varphi\|_{L^{2}(\widetilde{M})}^{2}}=(\Lambda+\varepsilon)^{2}
$$

which completes the proof of Theorem 1.2 .

\section{GENERIC METRICS AND HARMONIC SPINORS}

The alpha-genus is a ring homomorphism $\alpha_{*}: \Omega_{*}^{\text {spin }} \rightarrow K O^{-*}(\mathrm{pt})$ from the spin bordism ring to the real K-theory ring. This means that the alpha-genus $\alpha(M)$ of a spin manifold $M$ depends only on the spin bordism class of $M$ and that it is additive with respect to disjoint union and multiplicative with respect to product of spin manifolds. Choosing generators we have

$$
K O^{-n}(\mathrm{pt})=\left\{\begin{array}{cll}
\mathbb{Z} & \text { if } n \equiv 0,4 \bmod 8 \\
\mathbb{Z} / 2 & \text { if } n \equiv 1,2 \bmod 8 \\
0 & \text { otherwise } &
\end{array}\right.
$$

In this identification $\alpha_{8 k}=\widehat{A}_{8 k}$ and $\alpha_{8 k+4}=\frac{1}{2} \widehat{A}_{8 k+4}$ where $\widehat{A}_{*}: \Omega_{*}^{\text {spin }} \rightarrow \mathbb{Z}$ is the $\widehat{A}$-genus.

There is a generalization $\mathcal{D}$ of the Dirac operator called the $C l_{n}$-linear Atiyah-Singer operator [15]. This operator acts on sections of a bundle associated to the spin structure with fiber the Clifford algebra $C l_{n}$ and commutes with the right action of $C l_{n}$ on this bundle. The operator $\mathcal{D}$ has a "Clifford index" $\operatorname{ind}_{n}(\mathcal{D}) \in K O^{-n}(\mathrm{pt})$. This bundle of Clifford algebras decomposes into a sum of copies of the spinor bundle and the Clifford index is related to the index of the Dirac operator acting on sections of the spinor bundle as follows.

$$
\operatorname{ind}_{n}(\mathcal{D})=\left\{\begin{array}{ccc}
\operatorname{ind}\left(D^{+}\right) & \text {if } n \equiv 0 & \bmod 8, \\
\frac{1}{2} \operatorname{ind}\left(D^{+}\right) & \text {if } n \equiv 4 & \bmod 8, \\
\operatorname{dimker} D \bmod 2 & \text { if } n \equiv 1 & \bmod 8, \\
\operatorname{dim} \operatorname{ker} D^{+} \bmod 2 & \text { if } n \equiv 2 & \bmod 8 .
\end{array}\right.
$$


The index theorem for $\mathcal{D}$ states that $\operatorname{ind}_{n}(\mathcal{D})$ coincides with $\alpha_{n}(M)$ and this gives a lower bound on the dimension of the kernel of the Dirac operator $D$ on a manifold $M$,

$$
\operatorname{dim} \operatorname{ker} D \geq\left\{\begin{array}{cl}
|\widehat{A}(M)| & \text { if } n \equiv 0,4 \bmod 8, \\
|\alpha(M)| & \text { if } n \equiv 1 \bmod 8, \\
2|\alpha(M)| & \text { if } n \equiv 2 \bmod 8 .
\end{array}\right.
$$

The left hand side of this inequality depends on the Riemannian metric while the right hand side is a differential topological invariant. In the following definition we give a name to those Riemannian metrics for which equality holds.

Definition 3.1. A Riemannian metric $g$ on a compact spin manifold $M$ is called $D$ minimal if the kernel of the Dirac operator $D_{g}$ is no larger than it is forced to be by the index theorem.

In dimensions $3,5,6,7 \bmod 8$ the index of $D$ always vanishes and a metric $g$ is $D$ minimal if $\operatorname{ker} D_{g}=0$.

Denote by $h_{g}$ the dimension of the kernel of the Dirac operator $D_{g}$ and if the dimension is even denote by $h_{g}^{+}, h_{g}^{-}$the dimensions of the kernels of $D_{g}^{+}, D_{g}^{-}$. On an $n$-dimensional manifold $M$ with a $D$-minimal metric $g$ the numbers $h_{g}, h_{g}^{+}, h_{g}^{-}$are determined by $\alpha$ according to the following table.

\begin{tabular}{|c|c|c|}
\hline$n \bmod 8$ & $\alpha(M)$ & \\
\hline \hline \multirow{2}{*}{0,4} & $\geq 0$ & $h_{g}^{+}=\widehat{A}, \quad h_{g}^{-}=0$ \\
\cline { 2 - 3 } & $<0$ & $h_{g}^{+}=0, \quad h_{g}^{-}=-\widehat{A}$ \\
\hline \multirow{2}{*}{1} & 0 & $h_{g}=0$ \\
\cline { 2 - 3 } & 1 & $h_{g}=1$ \\
\hline \multirow{2}{*}{2} & 0 & $h_{g}^{+}=h_{g}^{-}=0$ \\
\cline { 2 - 3 } & 1 & $h_{g}^{+}=h_{g}^{-}=1$ \\
\hline $3,5,6,7$ & 0 & $h_{g}=0$ \\
\hline
\end{tabular}

With this terminology Conjecture B from the introduction can be phrased as

Conjecture B. On any compact connected spin manifold a generic metric is D-minimal.

The first result in this direction is by Anghel [1] who studies twisted Dirac operators on a manifold with a fixed Riemannian metric and shows that in dimensions $\leq 4$ a generic choice of connection on the twisting bundle gives a minimal kernel. The idea of the proof is to show that a non-minimal connection can be deformed to give nearby minimal connections. Such deformations are easily found only under the strong restriction on dimension. Using the formulas by Bourguignon and Gauduchon [6] for the variation of the spinor bundle and the Dirac operator with the Riemannian metric Maier [17] adapts the method of Anghel to prove Conjecture B in the same dimensions. 
Denote by $\mathcal{R}(M)$ the space of smooth Riemannian metrics on a compact spin manifold $M$ and by $\mathcal{R}_{\min }(M)$ the subset of $D$-minimal metrics. We have not yet made clear what we mean by the term "generic". Standard results from perturbation theory give the following proposition (see [12, Section VII.1.3], [1], [17]).

Proposition 3.2. $\mathcal{R}_{\min }(M)$ is open in the $C^{1}$-topology on $\mathcal{R}(M)$ and if it is nonempty it is dense in all $C^{k}$-topologies, $k \geq 1$.

We take "generic" to mean "in a set which is open in the $C^{1}$-topology on $\mathcal{R}(M)$ and dense in all $C^{k}$-topologies, $k \geq 1$ ". Denote by $\mathcal{M}_{\text {min }}$ the class of compact spin manifolds possessing at least one $D$-minimal metric. It follows from Proposition 3.2 that Conjecture $\mathrm{B}$ is true precisely for manifolds in $\mathcal{M}_{\min }$.

The rest of this paper is devoted to a study of the class $\mathcal{M}_{\min }$.

3.1. Properties of the class $\mathcal{M}_{\min }$. Note that the conclusion of Conjecture $\mathrm{B}$ may not hold if the manifold is not connected. Let $M$ be a manifold with $\alpha(M) \neq 0$ and let $-M$ be the same manifold with reversed orientation. Then the disjoint union $M+(-M)$ has $\alpha(M+(-M))=0$ but the kernel of the Dirac operator cannot be trivial because it then would be trivial also on $M$. The following proposition tells us that we can take disjoint unions (and later connected sums) in the class $\mathcal{M}_{\text {min }}$ whenever there is no cancellation of the alpha-genera.

Proposition 3.3. Let $M_{1}, M_{2} \in \mathcal{M}_{\min }$ with $\operatorname{dim} M_{1}=\operatorname{dim} M_{2}=n$. Suppose

1. $n \equiv 0,4 \bmod 8$ and $\alpha\left(M_{1}\right) \alpha\left(M_{2}\right) \geq 0$, or

2. $n \equiv 1,2 \bmod 8$ and $\alpha\left(M_{1}\right) \alpha\left(M_{2}\right)=0$, or

3. $n \equiv 3,5,6,7 \bmod 8$.

Then the disjoint union $M_{1}+M_{2} \in \mathcal{M}_{\text {min }}$.

Proof. We only treat the first case $n=4 k$ and $\alpha\left(M_{1}\right) \alpha\left(M_{2}\right) \geq 0$. The other cases are similar.

Let $g_{1}$ and $g_{2}$ be $D$-minimal metrics on $M_{1}$ and $M_{2}$ and let $g$ be the metric on $M_{1}+M_{2}$ given by $g_{1}$ and $g_{2}$. Suppose $\alpha\left(M_{1}+M_{2}\right)=\alpha\left(M_{1}\right)+\alpha\left(M_{2}\right)=0$, then $\alpha\left(M_{1}\right)=$ $\alpha\left(M_{2}\right)=0$ since we assume $\alpha\left(M_{1}\right) \alpha\left(M_{2}\right) \geq 0$. This means that $h_{g_{1}}=h_{g_{2}}=0$ so $h_{g}=0$ and $g$ is $D$-minimal. Next suppose $\alpha\left(M_{1}+M_{2}\right)=\alpha\left(M_{1}\right)+\alpha\left(M_{2}\right)>0$. Then we cannot have $\alpha\left(M_{1}\right) \leq 0$ and $\alpha\left(M_{2}\right) \leq 0$. By assumption $\alpha\left(M_{1}\right)$ and $\alpha\left(M_{2}\right)$ do not have different signs and we must have $\alpha\left(M_{1}\right) \geq 0$ and $\alpha\left(M_{2}\right) \geq 0$. Since $g_{1}$ and $g_{2}$ are $D$-minimal we have $h_{g}^{-}=h_{g_{1}}^{-}+h_{g_{2}}^{-}=0+0=0$ so $g$ is $D$-minmimal. The case $\alpha\left(M_{1}+M_{2}\right)<0$ is analogous to $\alpha\left(M_{1}+M_{2}\right)>0$.

A metric $g$ with positive scalar curvature is $D$-minimal since the Lichnerowicz formula forces $h_{g}=0$. This gives a rich source of manifolds in the class $\mathcal{M}_{\text {min }}$.

Proposition 3.4. Let $M$ be a compact spin manifold which has a metric of positive scalar curvature. Then $M \in \mathcal{M}_{\text {min }}$. 
A manifold with a positive scalar curvature metric has vanishing alpha-genus [10]. We will need a set of manifolds in $\mathcal{M}_{\text {min }}$ whose alpha-genera generate $K O^{-*}(\mathrm{pt})$.

Proposition 3.5. For each $n \equiv 0,1,2,4 \bmod 8, n \geq 1$, there is a $V_{n} \in \mathcal{M}_{\min }$ with $\operatorname{dim}\left(V_{n}\right)=n$ and $\alpha\left(V_{n}\right)=1$.

Proof. We prove this by induction starting with $n=1,2,4,8$.

Let $V_{1}$ be the circle with the spin structure which is not spin bordant to zero, let $V_{2}=$ $V_{1} \times V_{1}$, and let $V_{4}$ be the K3 surface. Let $V_{8}$ be a compact Riemannian 8-manifold with holonomy $\operatorname{Spin}(7)$ [11]. For $V_{1}$ we have $\alpha\left(V_{1}\right)=1$ and $h=1$ so $V_{1} \in \mathcal{M}_{\text {min }}$. By conformal invariance any metric $g$ on $V_{2}$ has $h_{g}^{+}=h_{g}^{-}=1$ and since $\alpha\left(V_{2}\right)=1$ we have $V_{2} \in \mathcal{M}_{\text {min }}$. The manifold $V_{4}$ has $\widehat{A}\left(V_{4}\right)=2 \alpha\left(V_{4}\right)=2$ so for any metric $g$ we have $h_{g}^{+} \geq 2$. Let $g_{0}$ be a Ricci flat metric on $V_{4}$. By the Lichnerowicz formula all harmonic spinors are parallel for this metric, so $h_{g_{0}}^{+} \leq \operatorname{rank}\left(\Sigma^{+} V_{4}\right)=2$. This means that $h_{g_{0}}^{+}=2$ so $g_{0}$ is $D$-minimal and $V_{4} \in \mathcal{M}_{\text {min }}$. The manifold $V_{8}$ with a $\operatorname{Spin}(7)$ holonomy metric $g_{8}$ has a one-dimensional space of parallel spinors [25] and since it is Ricci-flat there are no other harmonic spinors. This means that $\alpha\left(V_{8}\right)=\widehat{A}\left(V_{8}\right)=$ $1=h_{g_{8}}^{+}$so $g_{8}$ is $D$-minimal and $V_{8} \in \mathcal{M}_{\text {min }}$.

Next suppose that for some $n=8 k+1,8 k+2,4 k+4, k \geq 0$ we have a manifold $V_{n}$ with the desired properties. Set $V_{n+8}:=V_{8} \times V_{n}$ with the product metric $g_{n+8}=$ $g_{8}+g_{n}$, where $g_{n}$ is a $D$-minimal metric on $V_{n}$. Then $\alpha\left(V_{n+8}\right)=\alpha\left(V_{8}\right) \alpha\left(V_{n}\right)=1$. The spinor bundle on $V_{n+8}$ is isomorphic to the outer tensor product of the spinor bundles on $V_{8}$ and $V_{n}, \Sigma V_{n+8}=\Sigma V_{8} \widehat{\otimes} \Sigma V_{n}$, and the Dirac operators are related by $D_{g_{n+8}}^{2}=D_{g_{8}}^{2} \otimes 1+1 \otimes D_{g_{n}}^{2}$. From this we see that the harmonic spinors on the product are given precisely by products of harmonic spinors on the factors. In particular we have $h_{g_{n+8}}=h_{g_{8}} \cdot h_{g_{n}}=h_{g_{n}}$. Since $g_{n}$ is $D$-minimal $g_{n+8}$ is a $D$-minimal metric on $V_{n+8}$ and $V_{n+8} \in \mathcal{M}_{\text {min. }}$.

The most important step in our study of the class $\mathcal{M}_{\text {min }}$ is the following application of the surgery theorem for the Dirac spectrum.

Proposition 3.6. $\mathcal{M}_{\min }$ is closed under surgery in codimension $\geq 3$.

Proof. Let $M \in \mathcal{M}_{\text {min }}$ and suppose $\widetilde{M}$ is obtained from $M$ by surgery in codimension $\geq 3$. Let $g$ be a $D$-minimal metric on $M$ and choose $\Lambda>0$ so that the interval $[-\Lambda, \Lambda]$ does not contain any non-zero eigenvalues of $D_{g}$. By Theorem 1.2 there is a metric $\widetilde{g}$ on $\widetilde{M}$ with the property that $D_{\widetilde{g}}$ has precisely $h_{g}$ many eigenvalues in the interval $[-\Lambda, \Lambda]$ (counting multiplicity). Hence $h_{\widetilde{g}} \leq h_{g}$. Since surgery does not change the spin bordism class of a manifold we have $\alpha(\widetilde{M})=\alpha(M)$ and therefore $h_{\widetilde{g}} \geq h_{g}$ because $g$ is $D$-minimal. We conclude that $h_{\widetilde{g}}=h_{g}$ and therefore the metric $\widetilde{g}$ is also $D$-minimal.

3.2. Simply connected manifolds. We want to use Proposition 3.6 to construct new manifolds in the class $\mathcal{M}_{\text {min }}$. Without restrictions on the codimensions we can start with a spin manifold $N$ and do a sequence of surgeries ending up with a spin manifold $M$ if and only if $N$ and $M$ are spin bordant. The problem in our situation is that 
Proposition 3.6 allows only surgeries of codimension at least three. The same difficulty one encounters in the study of manifolds admitting metrics of positive scalar curvature. Gromov and Lawson [9] show that these surgeries are sufficient to handle simplyconnected manifolds (where in simply connected it is included that the manifold is connected).

Theorem 3.7. [9] Suppose that $M$ is a compact simply connected spin manifold of dimension $\geq 5$ and suppose that $M$ is spin bordant to a spin manifold $N$. Then $M$ can be obtained from $N$ by a sequence of surgeries of codimension $\geq 3$.

Using this theorem and the Surgery Theorem 1.2 we get the following result relating the spectrum of the Dirac operator for two ends of a spin bordism:

Theorem 3.8. Let $M$ be a compact simply connected spin manifold of dimension $\geq 5$ and let $M$ be spin bordant to a spin manifold $N$. Let $h$ be a Riemannian metric on $N$ and let $\Lambda, \varepsilon>0$ be such that $\pm \Lambda \notin \operatorname{spec}\left(D_{h}\right)$. Then there is a Riemannian metric $g$ on $M$ such that $D_{h}$ and $D_{g}$ are $(\Lambda, \varepsilon)$-spectral close.

We will now show that the manifolds from Propositions 3.4 and 3.5 are sufficiently plenty to allow us to find a manifold in $\mathcal{M}_{\text {min }}$ in each spin bordism class.

Proposition 3.9. Any compact spin manifold is spin bordant to a manifold in $\mathcal{M}_{\text {min }}$.

Proof. Let $M$ be a compact spin manifold of dimension $n$ and let $p=\alpha(M)$. If $p \geq 0$ let $p V_{n}$ be the disjoint union of $p$ copies of the manifold $V_{n}$ from Proposition 3.5 and if $p<0$ let $p V_{n}$ be the disjoint union of $-p$ copies of $-V_{n}$. We have $\alpha\left(M-p V_{n}\right)=$ $\alpha(M)-p \alpha\left(V_{n}\right)=0$ so by Theorem B of Stolz [24] $M-p V_{n}$ is spin bordant to a manifold $E$ which allows a positive scalar curvature metric. This means that $M$ is spin bordant to $E+p V_{n}$ which by Propositions 3.3 and 3.4 is in $\mathcal{M}_{\text {min }}$.

We are now ready to prove our main result on Conjecture B.

Theorem 3.10. Conjecture B is true for all simply connected spin manifolds of dimension at least five.

Proof. Suppose $M$ is a simply connected spin manifold with $\operatorname{dim} M \geq 5$. From the previous proposition we know that $M$ is spin bordant to a manifold in $\mathcal{M}_{\text {min }}$. Theorem 3.7 tells us that this bordism can be decomposed into a sequence of surgeries of codimension at least three. Using Proposition 3.6 we conclude that $M \in \mathcal{M}_{\min }$ and that the conjecture holds for $M$.

3.3. Non-simply connected manifolds. In the study of manifolds allowing positive scalar curvature metrics a machinery has been built up to analyse when two manifolds are related by surgeries of codimension $\geq 3$, see for example [19, 21]. We are now going to give one example of how results from this area can be used to prove Conjecture B for certain classes of fundamental groups.

For non-simply connected manifolds one uses a refinement of the ordinary spin bordism groups $\Omega_{*}^{\text {spin }}$. Let $\pi$ be a group and let $B \pi$ be a classifying space for $\pi$. The 
bordism groups $\Omega_{*}^{\text {spin }}(B \pi)$ consists of bordism classes of pairs $(M, f)$ where $M$ is a compact spin manifold and $f: M \rightarrow B \pi$ is a map. In particular, if $M$ has fundamental group $\pi$ we get such a pair $(M, f)$ since the universal cover of $M$ is a $\pi$-bundle over $M$ and corresponds to a map $f: M \rightarrow B \pi$.

In [18] Rosenberg proves the following generalization of the bordism theorem 3.7:

Theorem 3.11. Let $M$ be a compact spin manifold of dimension $\geq 5$ with fundamental group $\pi$. Let $f$ be the classifying map of the universal cover of $M$. Suppose that $(M, f)$ is equivalent to $\left(N, f^{\prime}\right)$ in $\Omega_{*}^{\text {spin }}(B \pi)$ (where $f^{\prime}$ is any map $N \rightarrow B \pi$ ). Then $M$ can be obtained from $N$ by a sequence of surgeries in codimension $\geq 3$.

For manifolds $M$ and $N$ satisfying the conditions of this theorem we get a bordism result for the Dirac spectrum just as Theorem 3.8.

A finite group $\pi$ is called periodic if it has periodic cohomology, that is if there is $d>0$ so that $H^{i}(\pi)=H^{i+d}(\pi)$ for all $i>0$. This includes cyclic groups of all orders.

Theorem 3.12. Suppose $M$ is a compact connected spin manifold with $\operatorname{dim} M \geq 5$ and fundamental group $\pi$ a periodic group of odd order. Then Conjecture B holds for $M$.

Proof. Let $f: M \rightarrow B \pi$ be the classifying map of the universal cover of $M$. The class $(M, f) \in \Omega_{*}^{\text {spin }}(B \pi)$ can be written as

$$
(M, f)=(M, f)-(M, \text { const })+(M, \text { const }) .
$$

Composition with the constant map $B \pi \rightarrow$ pt gives a map $\Omega_{n}^{\text {spin }}(B \pi) \rightarrow \Omega_{n}^{\text {spin }}(\mathrm{pt})$ and the first two terms $(M, f)-(M$, const $)$ constitute an element in the kernel $\widetilde{\Omega}_{n}^{\text {spin }}(B \pi)$ of this map. From Theorem 1.8 and fact (1.2) in [14] we know that this class can be represented by a manifold $M^{\prime}$ with positive scalar curvature and fundamental group $\pi$. Since $M^{\prime}$ has a metric of positive scalar curvature $M^{\prime} \in \mathcal{M}_{\text {min }}$. By doing surgery on embedded circles we can make the third term ( $M$, const) simply connected without changing its class in $\Omega_{*}^{\text {spin }}(B \pi)$. The resulting manifold $N$ is in $\mathcal{M}_{\text {min }}$ by Theorem 3.10 .

We thus have that $(M, f)$ is spin bordant to $\left(M^{\prime}, f^{\prime}\right)+(N$, const) over $B \pi$. Since $\alpha\left(M^{\prime}\right)=0$ Proposition 3.3 tells us that the disjoint union $M^{\prime}+N \in \mathcal{M}_{\min }$. Theorem 3.11 and Proposition 3.6 finish the proof.

Remark 3.13. The crucial point of the proof is the fact that for odd order periodic groups $\pi$ the kernel $\widetilde{\Omega}_{n}^{\text {spin }}(B \pi)$ can be represented by manifolds allowing positive scalar curvature metrics. This fact - and the theorem - holds for any odd order finite group for which the Gromov-Lawson-Rosenberg conjecture is true, see Section 3 of [20].

Remark 3.14. The torus $T^{n}$ with a flat metric (and the trivial spin structure) has a large space of harmonic spinors, even though $\alpha\left(T^{n}\right)=0$. It is not clear when in general the product of two manifolds in $\mathcal{M}_{\text {min }}$ is again in $\mathcal{M}_{\text {min }}$. By taking products we can however prove the following. 
On the torus $T^{n}$ with any spin structure there is a metric for which the kernel of the Dirac operator is trivial.

Namely, from the solution of Conjecture B for manifolds of low dimensions by Maier [17] we know that (for any spin structure) there is a metric $g_{3}$ on $T^{3}$ without nontrivial harmonic spinors. Write $T^{n}=T^{3} \times T^{n-3}$ and set $g_{n}=g_{3}+g_{n-3}$, where $g_{n-3}$ is any metric on $T^{n-3}$. Since $D_{g_{3}}$ does not have zero as an eigenvalue it follows from the Pythagorean formula for the eigenvalues of the product metric $g_{n}$ that $D_{g_{n}}$ has no zero eigenvalue.

\section{REFERENCES}

[1] N. Anghel, Generic vanishing for harmonic spinors of twisted Dirac operators, Proc. Am. Math. Soc. 124 (1996), 3555-3561.

[2] C. Bär, Lower eigenvalue estimates for Dirac operators, Math. Ann. 293 (1992), 39-46.

[3] _ Metrics with harmonic spinors, Geom. Func. Anal. 6 (1996), 899-942.

[4] A. L. Besse, Einstein manifolds, Springer-Verlag, Berlin-Heidelberg, 1987.

[5] B. Booß-Bavnbek and K. P. Wojciechowski, Elliptic boundary problems for Dirac operators, Birkhäuser, Boston Basel Berlin, 1993.

[6] J.-P. Bourguignon and P. Gauduchon, Spineurs, opérateurs de Dirac et variations de métriques, Comm. Math. Phys. 144 (1992), 581-599.

[7] J.-H. Eschenburg and E. Heintze, Comparison theory for Riccati equations, Manuscr. Math. 68 (1990), 209-214.

[8] T. Friedrich, Der erste Eigenwert des Dirac-Operators einer kompakten Riemannschen Mannigfaltigkeit nicht-negativer Krümmung, Math. Nachr. 97 (1980), 117-146.

[9] M. Gromov and H. B. Lawson, The classification of simply connected manifolds of positive scalar curvature, Ann. Math., II. Ser. 111 (1980), 423-434.

[10] N. Hitchin, Harmonic spinors, Adv. Math. 14 (1974), 1-55.

[11] D. D. Joyce, Compact 8-manifolds with holonomy Spin(7), Invent. Math. 123 (1996), 507-552.

[12] T. Kato, Perturbation theory for linear operators, second ed., Springer-Verlag, Berlin, 1976.

[13] D. Kotschick, Non-trivial harmonic spinors on generic algebraic surfaces, Proc. Am. Math. Soc. 124 (1996), 2315-2318.

[14] S. Kwasik and R. Schultz, Positive scalar curvature and periodic fundamental groups, Comment. Math. Helv. 65 (1990), no. 2, 271-286.

[15] H. B. Lawson and M.-L. Michelsohn, Spin geometry, Princeton University Press, Princeton, 1989.

[16] A. Lichnerowicz, Spineurs harmoniques, C. R. Acad. Sci. Paris 257 (1963), 7-9.

[17] S. Maier, Generic metrics and connections on spin- and $\operatorname{spin}^{c}$-manifolds, Commun. Math. Phys. 188 (1997), 407-437.

[18] J. Rosenberg, $C^{*}$-algebras, positive scalar curvature, and the Novikov conjecture, II, Geometric methods in operator algebras (Kyoto, 1983), Longman Sci. Tech., Harlow, 1986, pp. 341-374.

[19] J. Rosenberg and S. Stolz, Manifolds of positive scalar curvature, Algebraic topology and its applications, Math. Sci. Res. Inst. Publ. 27 (G. E. Carlsson et al., ed.), Springer-Verlag, New York, 1994, pp. 241-267.

[20] _ A "stable" version of the Gromov-Lawson conjecture, The Čech centennial (Boston, MA, 1993), Amer. Math. Soc., Providence, RI, 1995, pp. 405-418.

[21] Metrics of positive scalar curvature and connections with surgery, Surveys on surgery theory. Vol. 2. (S. Cappell et al., ed.), Princeton University Press, Princeton. Ann. Math. Stud. 149 (2001), 353-386

[22] E. Schrödinger, Diracsches Elektron im Schwerefeld I., Sitzungsber. Preuss. Akad. Wiss., Phys.Math. K1. (1932), 105-128.

[23] L. Seeger, Metriken mit harmonischen Spinoren auf geradedimensionalen Sphären, Dissertation, Universität Hamburg, 2000.

[24] S. Stolz, Simply connected manifolds of positive scalar curvature, Ann. Math., II. Ser. 136 (1992), 511-540. 
[25] McK. Y. Wang, Parallel spinors and parallel forms, Ann. Global Anal. Geom. 7 (1989), 59-68.

Universität Hamburg, FB Mathematik, Bundesstr. 55, 20146 Hamburg, Germany

E-mail address: baer@math.uni-hamburg.de, mattias.dahl@math.uni-hamburg.de 